\title{
Enlargement Of Left Ventricular Outflow Tract Using An Autologous Pericardial Patch For The Anterior Mitral Valve Leaflet And Septal Myectomy Through Trans-mitral Approach For The Treatment Of Hypertrophic Obstructive Cardiomyopathy
}

Hongqiang Zhang ( $\nabla$ zhang.hongqiang@zs-hospital.sh.cn )

Zhongshan Hospital Fudan University

\section{Kai Zhu}

Zhongshan Hospital Fudan University

\section{Fanshun Wang}

Zhongshan Hospital Fudan University

\section{Xiaoning Sun}

Zhongshan Hospital Fudan University

\section{Shouguo Yang}

Zhongshan Hospital Fudan University

Chunsheng Wang

Zhongshan Hospital Fudan University

\section{Research article}

Keywords: hypertrophic obstructive cardiomyopathy, septal myectomy, trans-mitral approach, autologous pericardial patch, surgical treatment

Posted Date: July 17th, 2020

DOl: https://doi.org/10.21203/rs.3.rs-43582/v1

License: (c) (i) This work is licensed under a Creative Commons Attribution 4.0 International License.

Read Full License

Version of Record: A version of this preprint was published at Journal of Cardiac Surgery on August 31st, 2021. See the published version at https://doi.org/10.1111/jocs.15950. 


\section{Abstract}

Background: Modified Morrow procedure is the gold standard of surgical intervention for hypertrophic obstructive cardiomyopathy (HOCM). However, there are certain cases without clear exposure through the traditional trans-aortic approach; we therefore described a trans-mitral approach by enlarging left ventricular outflow tract (LVOT) using an autologous pericardial patch for the anterior mitral valve leaflet and septal myectomy. We aimed to retrospectively analyze this series of patients to reveal its safety and efficiency.

Methods: We retrospectively analyzed 16 HOCM patients underwent enlargement of LVOT using an autologous pericardial patch for the anterior mitral valve leaflet and septal myectomy through transmitral approach in our center from January, 2016 to December, 2019. Baseline characteristics, operative details and postoperative data were extracted from our hospital medical records.

Results: Of the 16 patients, there was no operative mortality. No new onset atrial fibrillation, no new onset stroke with symptoms, no permanent pacemaker implantation and no ventricular septal defects formation were observed during operation and three months follow-up. The peak pressure gradient of LVOT decreased from $97.56 \pm 23.81 \mathrm{mmHg}$ to $7.56 \pm 2.13 \mathrm{mmHg}(P<0.01)$ after operation and $10.19 \pm 2.93$ $\mathrm{mmHg}(\mathrm{P}<0.01)$ three months after operation. The average aortic cross-clamp time was $54.56 \pm 6.10$ mins (range, 48 to 69 minutes). The systolic anterior motion (SAM) sign disappeared uneventfully in all cases. No patients had more than moderate MR.

Conclusions: Enlargement of LVOT using an autologous pericardial patch for the anterior mitral valve leaflet and septal myectomy through trans-mitral approach is feasible and reliable for the treatment of certain types of HOCM cases.

Trial registration: Not applicable.

\section{Background}

Hypertrophic cardiomyopathy is one of the most common heritable heart diseases with an incidence of about one in 500 individuals $[1,2]$. The morphologic and functional feature differs according to the site of the hypertrophic lesions, including the left and/or right ventricular. Hypertrophic obstructive cardiomyopathy (HOCM) is one form of hypertrophic cardiomyopathy with specific pathophysiological characteristic, i.e. the obstruction of the left ventricular outflow tract (LVOT) with/without concomitant mitral regurgitation (MR), caused by the thickened septal muscle and systolic anterior motion (SAM) of anterior mitral leaflet $[3,4,5]$.

For patients with obstruction of LVOT and/or mitral valve regurgitation, symptomatic signs just like dyspnea, chest pain and syncope are more likely occurred and these patients often do not respond adequately to drug therapy, such as beta-blockade, diltiazem, etc. Therefore, these patients remain significantly symptomatic and have a dissatisfactory long-term prognosis, which means high mortality 
and morbidity than hypertrophic cardiomyopathy patients without obstruction of LVOT $[2,6]$. Current invasive treatments include alcohol septal ablation and surgical myectomy, especially surgical myectomy of the thicken LVOT muscle has been approved to have the most satisfactory results $[7,8]$. For a long time, modified Morrow procedure has been the gold standard of surgical treatment, which was performed through the trans-aortic approach. In fact, in addition to modified Morrow operation through the transaortic approach, there are other approaches, including tans-apical and trans-mitral approach $[9,10,11,12$, 13]. In clinical practice, we found many special cases, such as the aortic valve annulus is very small, the angle between the aortic valve annulus and the mitral valve annulus is less than 120 degrees, the anterior mitral leaflet is too long, etc. It is difficult for these cases to completely resect the hypertrophic LVOT muscle through the tans-aortic approach. Thus, we prefer trans-mitral approach to perform septal myectomy and mitral valves repair. In this study,we aimed to review the results and experiences of these cases in our centre.

\section{Materials And Methods}

\section{Patients and characteristics}

Between January 2016 and December 2019, a total of 16 consecutive HOCM patients underwent surgical enlargement of the LVOT using an autologous pericardial patch and septal myectomy through transmitral approach in our centre were recruited. Baseline characteristics were extracted from our hospital medical records. In this case series, 7 of them were male, the average age was $47.94 \pm 12.79$ years. All patients were diagnosed via transthoracic echocardiography (TTE) before operation and transesophagel echocardiography (TEE) during operation. Preoperative and postoperative peak LVOT gradient, SAM sign, MR grade and maximum thickness of septal were measured for each patient to evaluate surgical results. Postoperative TTE was performed for each patient three months after the operation.

The surgical indication for these patients is the LVOT peak pressure gradient $>50 \mathrm{mmHg}$ with/without MR and symptomatic due to failed drug therapy. Patients indicated for modified Morrow Procedure through trans-aortic approach were excluded from this series. Aortic cross-clamp time and postoperative complications were collected to evaluate surgical safety.

This study was approved by the ethics committee of Zhongshan Hospital Fudan University, all patients gave their informed consent.

\section{Operative technique}

The surgery was conventional median sternotomy. Ascending aorta, superior and inferior vena cava were cannulated, after initiation of the cardiopulmonary bypass, aorta was cross-clamped and cold blood cardioplegia was introduced. We preferred a trans-right atrium and atrial septum approach to expose the anterior leaflet of mitral valve. The incision of mitral valve was at the root of the anterior leaflet, about 2-5 $\mathrm{mm}$ to the annulus, leaving an edge to repair. It usually carried from the right fibrous trigone to the left without freeing the whole anterior leaflet. After this incision, the anterior leaflet attachments would be 
released, and the ventricular septum and bottom of the anterior papillary muscle could be exposed clearly. Septal muscle myectomy was carried from the nadir of right aortic valve sinus to the left fibrous trigone, papillary muscle attachments were released at the same time. The depth of septal muscle resection was measured by TEE before operation and mainly depended on surgeons' experiences. The resection should be carefully carried to avoid injuring the aortic valve, atrioventricular and interventricular conduction beam. After septal myectomy, the anterior leaflet was reattached to the residual leaflet rim by using an prepared fusiform autologous pericardial patch ( $1 \%$ of glutaraldehyde solution, $10 \mathrm{mins}$ ). The length of the patch could be measured according to the incision of the leaflet and the maximum width of the patch was usually about $10-15 \mathrm{~mm}$ to make sure allowing the leaflet billowing away from the septum and enlarging the LVOT completely. At last we usually used a flexible band annuloplasty to increase the height of the leaflet coaptation in order to avoid the recurrence of MR.

\section{Statistical Analysis}

Continuous variables were expressed as mean \pm standard error of the mean. Student $t$ test was used to deal with group comparisons for analyses of continuous variables.

Statistics were performed with Stata 12.1 (Stata Corp, College Station, TX). A p value less than 0.05 was considered as the criterion for statistical significance.

\section{Results}

\section{Baseline characteristics}

Of the 16 patients recruited in this series, 7 were male, the mean age of the series was $47.94 \pm 12.79$ years (range, 16 to 65 years). The baseline characteristics were summarized in Table 1. All the patients recruited had severe symptoms and heart failure (NYHA II IV) refractory to regular drug therapy. Preoperative atrial fibrillation was found in $3(3 / 16,18.75 \%)$ patients. No one had an invasive treatment (including alcohol septal ablation and surgical septal myectomy) before. The baseline preoperative peak pressure gradient of LVOT was $97.56 \pm 23.81 \mathrm{mmHg}$ (range, 70 to $156 \mathrm{mmHg}$ ), the maximum thickness of septum was $18.38 \pm 3.56 \mathrm{~mm}$ (range, 14 to $27 \mathrm{~mm}$ ), SAM sign was observed in all patients by TEE before operation. 15 of the patients had more than moderate MR, only 1 patient had mild MR.

\section{Perioperative results}

There was no patient died in this series, the overall mortality rate of $0 \%$. No new onset atrial fibrillation or permanent pacemaker implantation was observed before being discharged from hospital in our study, new onset LBBB was observed in 2 patients. No new onset stroke with symptoms was found during hospital stay. The TEE examination intraoperatively revealed no ventricular septal defects, the peak pressure gradient of LVOT decreased from $97.56 \pm 23.81 \mathrm{mmHg}$ to $7.56 \pm 2.13 \mathrm{mmHg}(P<0.01) \otimes F i g 1 \rrbracket$. The average aortic cross-clamp time was $54.56 \pm 6.10$ mins (range, 48 to 69 minutes). The SAM sign disappeared uneventfully in all cases according to TEE examination during operation, almost all the 
patients had no more than mild MR, except for one who had mild to moderate MR. Of all the patients, 3 had a small aortic annulus/root, 6 patients had a small aortic and mitral annulus plane angle less than 120 degrees. 7 patients had anterior mitral valve lesions, and 4 of them combined posterior mitral valve lesion. Details were shown in Table 2.

\section{Follow-up results}

All patients underwent routine TTE examination three months after the operation. The mean peak pressure gradient of LVOT was $10.19 \pm 2.93 \mathrm{mmHg} 3$ months after the operation. No more MR was detected compared to the discharge status. One patient rehospitalized for pericardial effusion and recovered uneventfully after receiving pericardiocentesis and drainage.

\section{Discussion}

Hypertrophic obstructive cardiomyopathy ( $\mathrm{HOCM})$ is a common hereditary heart disease with nonspecific symptoms, just like dyspnea, chest pain and syncope on exercise $[1,2,6]$. For patients without relieving symptoms after regularly drug treatment, surgical intervention is required. In this retrospective study, we described a trans-mitral approach through the base of anterior mitral valve leaflet, with a special way to reattach the anterior mitral valve leaflet, for some special types of HOCM patients. The results confirmed that using this special technique to treat HOCM patients in this series is feasible and reliable.

Although there are many different surgical approaches and methods, the modified Morrow procedure through trans-aortic approach is still considered as the gold standard surgical treatment, showing a verified surgical result and good remission of symptoms for a long time $[8,9,11]$. The trans-mitral approach was firstly described in 1963 by Lillehei and Levy [10]. They chose this approach for HOCM patients just because of suboptimal exposure through the traditional trans-aortic approach. As we all know, a satisfactory procedure for HOCM consists of three major goals: completely thickened septal muscle resection, dealing with the anterior mitral valve leaflet in avoid of postoperative SAM, and release of abnormal papillary muscle and chordae attachments $[8,9,11,12,13]$. Compared with the traditional trans-aortic approach via the aortic incision, the trans-mitral approach via the base of the anterior mitral leaflet provided a more clear panoramic exposure of the interventricular septum, making myectomy easily and avoiding heart block $[14,15]$, through this approach, experienced surgeons could even identify the pale fibrous hinges subaortic hit caused by $\operatorname{SAM}[16,17,18]$. Also this approach provided a wide vision of the anterior papillary muscles and its base. This allowed surgeons completely address mitral valve abnormalities, release any abnormal papillary muscle and chordae insertion in avoid of postoperative SAM simultaneously $[16,17,18]$. According to the literatures $[3,4,5]$, more than half of the HOCM patients suffered from mitral valve regurgitation accompanied with obstruction of LVOT. Therefore, the trans-mitral approach enables surgeons to deal with this situation at the same time especially for patients with structural abnormality of the mitral valve.

Since all the patients in our study do not suffer from severe structural abnormalities of the mitral valve, our choice is to repair the valve instead of valve replacement. There are different mitral valve incisions 
according to literatures. Surgeons from University of Leipzig described a trans-mitral approach by repairing the valve with implantation of neochordae and annuloplasty $[12,16]$. They cut all native chords connected to the anterior mitral leaflet to expose the septum by pulling up the free leaflet and replacement of the anterior mitral leaflet chords using the Loop Technique. It really works for some cases, but not for the patients with a small angle between the aortic valve annulus and the mitral valve annulus plane, therefore we preferred the technique described by W. Randolph Chitwood $[17,18]$ and make some modifications for our cases. Dr. Chitwood made the mitral valve incision at the base of the anterior mitral valve leaflet and re-suspended it using a 4 - 0 PTFE suture, they interposed a pericardial patch between the annulus and cut leaflet edge when the anterior leaflet has been shortened. However, for these specific cases in our study, isolated myectomy often could not relieve LVOT obstruction and mitral valve SAM completely, in avoiding of mitral valve replacement and postoperative SAM, we reattach the anterior mitral valve leaflet to the residual leaflet rim by using an prepared fusiform autologous pericardial patch in all cases. We strongly recommend that the width of the patch should be as wide as possible to ensure that the root of the anterior mitral valve leaflet is sufficiently billowing away from the LVOT during the systolic period. The LVOT obstruction and mitral valve SAM were completely resolved during follow up in our cases, with no severe complications after operation and no occurrence MR.

The limitation of our study was the small-sized patient population and lack of long-term follow up since the cohort of cases were rare. For each case, we planted a flexible band annuloplasty to increase the height of the leaflet coaptation in order to avoid the recurrence MR, but the long-term prognosis accompany with the pericardial patch getting calcification is unknown.

Although modified Morrow procedure is still the gold standard surgical intervention for HOCM, enlargement of LVOT using an autologous pericardial patch for the anterior mitral valve leaflet and septal myectomy through trans-mitral approach is feasible and reliable for certain cases. Since the pathology of different HOCM forms varies in patients, personalized procedure plan should be prepared and the one in our study could be a proper choice.

\section{Conclusions}

Enlargement of LVOT using an autologous pericardial patch for the anterior mitral valve leaflet and septal myectomy through trans-mitral approach is feasible and reliable for the treatment of certain types of HOCM cases.

\section{List Of Abbreviations}

HOCM: hypertrophic obstructive cardiomyopathy

LVOT: left ventricular outflow tract

MR: mitral regurgitation 
SAM: systolic anterior motion

TTE: transthoracic echocardiography

TEE: transesophagel echocardiography

\section{Declarations}

\section{Ethics approval and consent to participate}

This study was approved by the ethics committee of Zhongshan Hospital Fudan University, all patients gave their informed consent.

\section{Consent for publication}

Not applicable.

\section{Availability of data and materials}

The datasets used and analysed during the current study are available from the corresponding author on reasonable request.

\section{Competing interests}

The authors declare that they have no competing interests.

\section{Funding}

Not applicable.

\section{Authors' contributions}

Hongqiang Zhang, Kai Zhu and Fanshun Wang conceived and designed the study, collected and analysed data. Hongqiang Zhang and Kai Zhu wrote the paper. Xiaoning Sun and Shouguo Yang helped perform the analysis with constructive discussions. Chunsheng Wang performed all the procedures. Hongqiang Zhang and Chunsheng Wang reviewed and edited the manuscript. All authors read and approved the final manuscript.

\section{Acknowledgements}

Not applicable.

\section{Authors' information}

Department of Cardiac Surgery, Zhongshan Hospital Fudan University 


\section{Footnotes}

Not applicable.

\section{References}

1. Kramer CM, Appelbaum E, Desai MY, Desvigne-Nickens P, DiMarco JP, Friedrich MG, et al. Hypertrophic Cardiomyopathy Registry: The rationale and design of an international, observational study of hypertrophic cardiomyopathy. Am Heart J. 2015;170(2):223-30.

2. Maron BJ, Towbin JA, Thiene G, Antzelevitch C, Corrado D, Arnett D, et al. Contemporary definitions and classification of the cardiomyopathies: an American Heart Association Scientific Statement from the Council on Clinical Cardiology, Heart Failure and Transplantation Committee; Quality of Care and Outcomes Research and Functional Genomics and Translational Biology Interdisciplinary Working Groups; and Council on Epidemiology and Prevention. Circulation. 2006;113(14):1807-16.

3. Hong JH, Schaff HV, Nishimura RA, Abel MD, Dearani JA, Li Z, et al. Mitral regurgitation in patients with hypertrophic obstructive cardiomyopathy: implications for concomitant valve procedures. J Am Coll Cardiol. 2016;68(14):1497-504.

4. Wehman B, Ghoreishi M, Foster N, Wang L, D'Ambra MN, Maassel N, et al. Transmitral Septal Myectomy for Hypertrophic Obstructive Cardiomyopathy. Ann Thorac Surg. 2018;105(4):1102-08.

5. Kaple RK, Murphy RT, DiPaola LM, Houghtaling PL, Lever HM, Lytle BW, et al. Mitral valve abnormalities in hypertrophic cardiomyopathy: echocardiographic features and surgical outcomes. Ann Thorac Surg. 2008;85(5):1527-35.

6. Maron BJ, Rowin EJ, Casey SA, Link MS, Lesser JR, Chan RH, et al. Hypertrophic Cardiomyopathy in Adulthood Associated With Low Cardiovascular Mortality With Contemporary Management Strategies. J Am Coll Cardiol. 2015;65(18):1915-28.

7. Sen-Chowdhry S, Jacoby D, Moon JC, McKenna WJ. Update on hypertrophic cardiomyopathy and a guide to the guidelines. Nat Rev Cardiol. 2016;13(11):651-75.

8. Redaelli M, Poloni CL, Bichi S, Esposito G. Modified surgical approach to symptomatic hypertrophic cardiomyopathy with abnormal papillary muscle morphology: Septal myectomy plus papillary muscle repositioning. J Thorac Cardiovasc Surg. 2014;147(5):1709-11.

9. Binaco I, Spirito P, Poggio D, Casati V, Grillo M, Ferrazzi P. Transaortic mitral valve secondary chordal cutting in patients with obstructive hypertrophic cardiomyopathy and mild septal hypertrophy. Ann Cardiothorac Surg. 2017;6(4):426-8.

10. Lillehei CW, Levy MJ. Transatrial exposure for correction of subaortic stenosis. JAMA. 1963;86:8-13.

11. Ralph-Edwards A, Vanderlaan RD, Bajona P. Transaortic septal myectomy: Techniques and pitfalls. Ann Cardiothorac Surg. 2017;6(4):410-5. 
12. Meier S, Noack T, Mohr FW, Seeburger J, Passage J. Transmitral myectomy and how to deal with systolic anterior motion (SAM) in hypertrophic obstructive cardiomyopathy. Ann Cardiothorac Surg. 2017;6(4):416-8.

13. Kotkar KD, Said SM, Schaff HV. Transapical approach for myectomy in hypertrophic cardiomyopathy. Ann Cardiothorac Surg. 2017;6(4):419-22.

14. Khalpey Z, Korovin L, Chitwood WR Jr, Poston R. Robot-assisted septal myectomy for hypertrophic cardiomyopathy with left ventricular outflow tract obstruction. J Thorac Cardiovasc Surg. 2014;147(5):1708-9.

15. Dulguerov F, Marcacci C, Alexandrescu C, Chan KM, Dreyfus GD. Hypertrophic obstructive cardiomyopathy: the mitral valve could be the key. Eur J Cardiothorac Surg. 2016;50(1):61-5.

16. Mohr FW, Seeburger J, Misfeld M. Keynote lecture transmitral hypertrophic obstructive cardiomyopathy (HOCM) repair. Ann Cardiothorac Surg. 2013;2(6):729-32.

17. Chitwood WR. Robotic trans-atrial and trans-mitral ventricular septal resection. Ann Cardiothorac Surg. 2017;6(1):54-9.

18. Chitwood WR. Idiopathic hypertrophic subaortic septal obstruction: Robotic trans-atrial and transmitral ventricular septal resection. Oper Tech Thorac Cardiovasc Surg. 2012;17:251-60.

\section{Tables}




\begin{tabular}{|c|c|}
\hline \multicolumn{2}{|l|}{ Table 1 Baseline characteristics } \\
\hline Preoperative Variables & Values \\
\hline Gender(Male/Female) & $7 / 9$ \\
\hline Age, years & $47.94 \pm 12.79$ \\
\hline Atrial fibrillation(y/n) & $3 / 13$ \\
\hline Stroke(y/n) & $1 / 15$ \\
\hline Pacemaker(y/n) & $1 / 15$ \\
\hline \multicolumn{2}{|l|}{ NYHA functional class } \\
\hline II & 2 \\
\hline III & 13 \\
\hline IV & 1 \\
\hline LVEF, \% & $63.00 \pm 2.94$ \\
\hline Septal thickness, mm & $18.38 \pm 3.56$ \\
\hline LVOT Peak gradient, mm Hg & $97.56 \pm 23.81$ \\
\hline SAM & 16 \\
\hline \multicolumn{2}{|l|}{ Mitral Regurgitation } \\
\hline mild & 1 \\
\hline moderate & 10 \\
\hline severe & 5 \\
\hline \multicolumn{2}{|c|}{$\begin{array}{l}\text { LVEF }=\text { Left ventricular ejection fraction } \\
\text { LVOT = Left ventricular outflow tract } \\
\text { NYHA = New York Heart Association }\end{array}$} \\
\hline
\end{tabular}




\begin{tabular}{|ll|}
\hline Table 2 Summary of operative details & \\
\hline Operative Details & Values \\
\hline Mortality & 0 \\
\hline New onset Stroke & 0 \\
\hline New onset Heart Block & \\
\hline LBBB & 2 \\
\hline AVB & 0 \\
\hline New onset atrial fibrillation & 0 \\
\hline Left ventricular outflow gradient, mm Hg & $7.56 \pm 2.13$ \\
\hline $\begin{array}{l}\text { Pathology } \\
\text { Small aortic annulus/root } \\
\text { Small aortic and mitral annulus angle }\end{array}$ & 3 \\
Combined mitral valve lesion \\
Anterior & 7 \\
\hline Mosterior & 4 \\
\hline Mitral regurgitation & \\
\hline Trace & \\
\hline Mild & 4 \\
\hline Mild to Moderate & 10 \\
\hline SAM & 2 \\
\hline Cross-clamp time, minutes & 0 \\
\hline
\end{tabular}

\section{Figures}




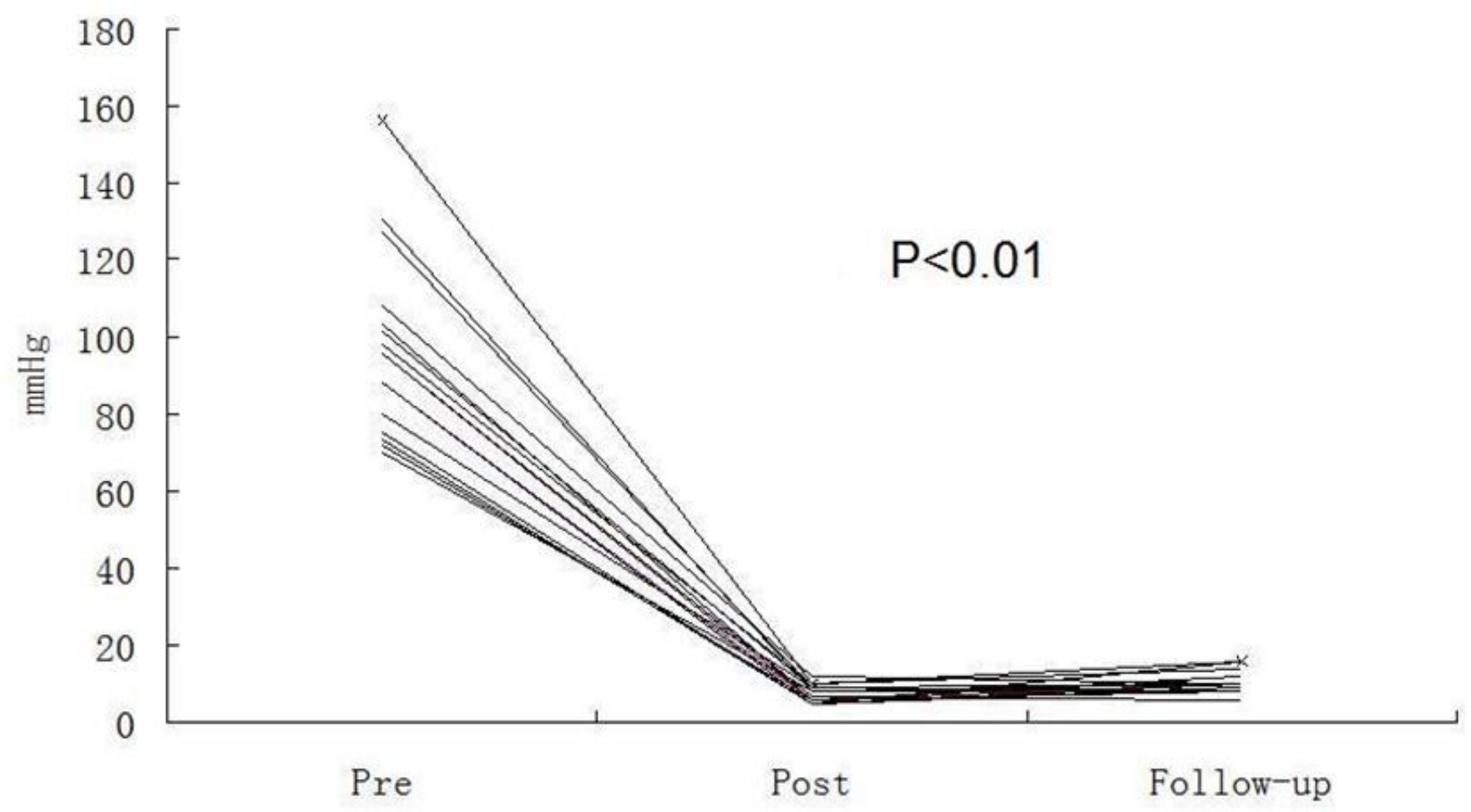

\section{Figure 1}

The LVOT peak gradient of preoperative (Pre), postoperative (Post) and three months after operation (Follow-up) significantly decreased from $97.56 \pm 23.81 \mathrm{mmHg}$ to $7.56 \pm 2.13 \mathrm{mmHg}(p<0.001)$ and $10.19 \pm 2.93 \mathrm{mmHg}$. 